\title{
Analytical Solution of Plastoelastic Buckling of Rectangular Section Column Strengthened by Carbon Fibre Sheets
}

\author{
Chengzhu Qiu ${ }^{1, a}$ \\ ${ }^{1}$ Civil Engineering Academy of Liaoning Science and Technology University, Anshan,China \\ acivilqiu@126.com
}

Key words: member; carbon fibre; bending moment; buckling

Abstract.Based on the Ježek method of computing elastic-plastic buckling of compression-bending member, analytical solutions of buckling ultimate load for rectangular column strengthened by carbon fibre sheets are derived. A new method of theoretical research on elastic-plastic buckling of steel member is provided. Some conclusions are obtained by analyzing the stress of rectangular column before and after being pasted with carbon fiber sheets. The effects of different thickness of composites on the ultimate load of rectangular column are discussed, the theoretical analysis is proved correct by the numerical simulation test.

\section{Introduction}

The plastic bearing capacity of steel members strengthened by carbon fibre sheets can be improved, It is necessary to study the plastic instability state of steel structure pasted with carbon fiber sheets. Analytical expressions of ultimate load of buckling about neutral axis with maximum moment of inertia for rectangular column that its upper and lower surfaces are pasted with carbon fibre sheets are derived, it is confirmed that carbon fibre sheets is effective to enhance the plastic buckling of steel members.

Unilateral or bilateral plastic zones may appear in the tension and compression zone of cross section of compression-bending member ${ }^{[1]}$, simply supported structure at both ends with plastic zone only in compression-side is discussed in the paper.

\section{Elastic-plastic buckling formula for column with plastic zone only in compression-side}

Formula derivation under these basic assumptions: (1)The member is only subjected to axial compressive load $\mathrm{P}$ and bending moment $\mathrm{M}$, both ends are hinged(Fig.1); (2)The member is an ideal elastic plastic body ${ }^{[2]}$; (3) Carbon fibre has brittle elastic constitutive relation and always maintains good line elasticity before being broke; (4)Carbon fibre is affixed to the upper and lower surfaces of the member, both are well bonded and no relative slip; (5)The bending deformation of the member is half wave sine curve; (6)The member deformation is a small deformation; (7) Bending occurs only on the strong axis of the member ${ }^{[3]}$.

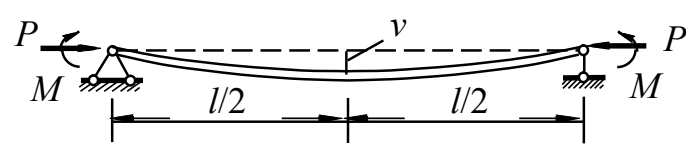

Fig. 1 Simple supported beam under axial compressive load and bending moment 
Now, $P_{y}=A \sigma_{y}$ expresses full section yield pressure of the member under axial compressive load, According to the balance condition of the axial force of the member section ${ }^{[4]}$,

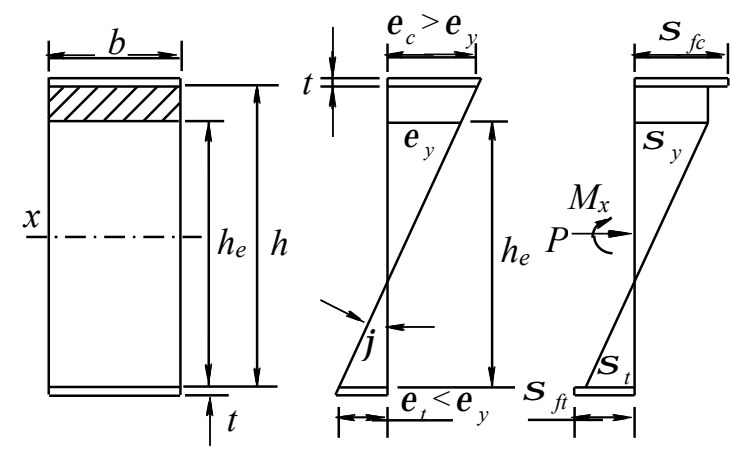

Fig. 2 The central section just bears the strain and stress of the compressive yield zone

$$
P=\sigma_{y} A-\frac{1}{2}\left(\sigma_{y}+\sigma_{t}\right) b h_{e}+E_{f}\left(\varepsilon_{c}-\varepsilon_{t}\right) b t
$$

In the equation, $\sigma_{y}$ is member yield strength; $A$ is cross sectional area of member; $\sigma_{t}$ is lower surface stress of the member, less than $\sigma_{y} ; h_{\mathrm{e}}$ is elastic core height of member cross section (Fig. 2, shadow part is plastic zone ) ; $E_{\mathrm{f}}$ is elastic modulus of carbon fibre; $\varepsilon_{c}$ is the strain of upper surface of member; $\varepsilon_{t}$ is the strain lower surface of member and carbon fibre ${ }^{[5]} ; t$ is the thickness of carbon fibre.

From Fig.2,

$$
\varepsilon_{c}=\frac{h}{h_{e}}\left(\varepsilon_{y}+\varepsilon_{t}\right)-\varepsilon_{t}
$$

Where $\varepsilon_{y}$ is yield strain。

Put Eq. (2) into Eq.(1), we get

$$
P=P_{y}-\frac{1}{2}\left(\sigma_{y}+\sigma_{t}\right) b h_{e}+\frac{E_{f}}{E}\left[\frac{h}{h_{e}}\left(\sigma_{y}+\sigma_{t}\right)-2 \sigma_{t}\right] b t
$$

According to the balance condition of moment,

$$
M_{x}=M+P v=\frac{1}{2}\left(\sigma_{y}+\sigma_{t}\right) b h_{e}\left(\frac{h}{2}-\frac{h_{e}}{3}\right)+E_{f}\left(\varepsilon_{c}+\varepsilon_{t}\right) b t \frac{h}{2}
$$

Put Eq. (2) into Eq.(4), and according to $\varepsilon=\frac{\sigma}{E}$, then

$$
M+P v=\frac{1}{2}\left(\sigma_{y}+\sigma_{t}\right) b h_{e}\left(\frac{h}{2}-\frac{h_{e}}{3}\right)+\frac{E_{f}}{E} \frac{h}{h_{e}}\left(\sigma_{y}+\sigma_{t}\right) b t \frac{h}{2}
$$

From Fig.2, 


$$
\varphi=\frac{\varepsilon_{y}+\varepsilon_{t}}{h_{e}}=\frac{\sigma_{y}+\sigma_{t}}{E h_{e}}
$$

According to the hypothesis of deformation curve, deflection curve is

$$
y=v \sin \frac{\pi x}{l}
$$

Curvature of the central cross section is

$$
\varphi=-y^{\prime \prime}\left(\frac{l}{2}\right)=\frac{v \pi^{2}}{l^{2}}=\frac{\sigma_{y}+\sigma_{t}}{E h_{e}}
$$

From Eq.(8),

$$
\sigma_{t}=\frac{v \pi^{2}}{l^{2}} E h_{e}-\sigma_{y}
$$

Where,

$$
\kappa=\frac{\pi^{2}}{l^{2}} E, \lambda=\frac{E_{f}}{E}
$$

Then,

$$
\sigma_{t}=\kappa v h_{e}-\sigma_{y}
$$

Put Eq. (11) into Eq.(3) and (5),

$$
\begin{array}{r}
P=P_{y}-\frac{1}{2} \kappa v b h_{e}^{2}+\lambda \kappa v b t h-2 \lambda \kappa v b t h_{e}+2 \lambda \sigma_{y} b t \\
M+P v=\frac{1}{2} \kappa v b h_{e}^{2}\left(\frac{h}{2}-\frac{h_{e}}{3}\right)+\lambda \kappa v b t \frac{h^{2}}{2}
\end{array}
$$

Eq.(12) is a quadratic equation with one unknown, we get

$$
h_{e}=-2 \lambda t+2 \lambda t \sqrt{1+\frac{h}{2 \lambda t}+\frac{\sigma_{y}}{\lambda \kappa v t}-\frac{P-P_{y}}{2 \lambda^{2} \kappa v b t^{2}}}
$$

Take the derivative of $\mathrm{v}$ in Eq.(14) and use the extremal condition $\frac{\mathrm{d} P}{\mathrm{~d} v}=0$,

$$
\frac{\mathrm{d} h_{e}}{\mathrm{~d} v}=\frac{P-P_{y}-2 \lambda \sigma_{y} b t}{\sqrt{4 \lambda^{2} \kappa^{2} v^{4} b^{2} t^{2}+2 \lambda \kappa^{2} v^{4} b^{2} h t+4 \lambda \kappa \sigma_{y} v^{3} b^{2} t-2 \kappa v^{3} b\left(P-P_{y}\right)}}
$$

Then take the derivative of $\mathrm{v}$ in Eq.(13),

$$
P=\frac{1}{2} \kappa b h_{e}^{2}\left(\frac{h}{2}-\frac{h_{e}}{3}\right)+\frac{1}{2} \kappa v b\left(h h_{e}-h_{e}^{2}\right) \frac{\mathrm{d} h_{e}}{\mathrm{~d} v}+\lambda \kappa b t \frac{h^{2}}{2}
$$

Take Eq.(14) into Eq.(13), take Eq.(14)and (15)into Eq.(16), so we can obtain two equations of $P \sim v$ relationship, we can draw up their relation curve in the same coordinate system, then their 
intersection is ultimate load of compression-flexure member.

\section{Calculation example}

One steel column of rectangular section, cross section width $b=100 \mathrm{~mm}$, section height $h=200 \mathrm{~mm}$, elastic modulus $E=206 \times 10^{9} \mathrm{~Pa}$, material yield limit $\sigma_{\mathrm{y}}=235 \times 10^{6} \mathrm{~Pa}$, Elastic modulus of composite $E_{\mathrm{f}}=235 \times 10^{9} \mathrm{~Pa}$, paste thickness $t=0.835 \mathrm{~mm}$.

According to equation $l_{0}=\pi \sqrt{E I / \sigma_{y} A}$, critical length $l_{0}=5.37 \mathrm{~m}$ is acquired, Elastic limit moment $M_{e}=\sigma_{y} W=0.157 \times 10^{6} \mathrm{Nm}$, plastic limit bending moment $M_{u}=1.5 M_{e}=0.235 \times 10^{6} \mathrm{Nm}$.

Through table1 and table2, the enhancement of composites becomes greater and the plastic zone of steel member becomes smaller with the increasing of bending moment,it indicates that the plastic bearing capacity of steel member pasted with composites can be enhanced. In addition, the maximum stress of composites only is hundreds Mpa and far less than the breaking strength of composites.

Table1 Ultimate load before and after pasting composites $\mathrm{Pu}(\mathrm{KN})$

\begin{tabular}{|l|l|l|l|l|}
\hline$M\left(\times 10^{6}\right)$ & 0.01 & 0.05 & 0.1 & 0.15 \\
\hline $\begin{array}{l}\text { before pasting } \\
\text { carbon fiber }\end{array}$ & 1899 & 1311 & 845 & 444 \\
\hline $\begin{array}{l}\text { After pasting } \\
\text { carbon fiber }\end{array}$ & 1957 & 1364 & 898 & 500 \\
\hline rise (\%) & 3.1 & 4.0 & 6.3 & 12.6 \\
\hline
\end{tabular}

Table2 Elastic core height before and after pasting composites he $(\mathrm{mm})$

\begin{tabular}{|l|l|l|l|l|}
\hline$M\left(\times 10^{6}\right)$ & 0.01 & 0.05 & 0.1 & 0.15 \\
\hline $\begin{array}{l}\text { before pasting } \\
\text { carbon fiber }\end{array}$ & 193 & 170 & 108 & 70.6 \\
\hline $\begin{array}{l}\text { After pasting } \\
\text { carbon fiber }\end{array}$ & 193 & 170 & 147 & 118 \\
\hline add (\%) & 0.0 & 0.0 & 36.1 & 67.1 \\
\hline
\end{tabular}

As is shown in Fig.3, there is a basic linear relationship between the thickness of composites pasted on member and the increasing of ultimate load of steel member.

In order to prove the correctness of the theoretical calculation formula, $P_{\mathrm{u}}-M$ curve obtained by theoretical calculation formula and computing result obtained by the finite element software ANSYS were compared(see Fig.4), it is testified that theoretical calculation method and numerical solution are very consistent.

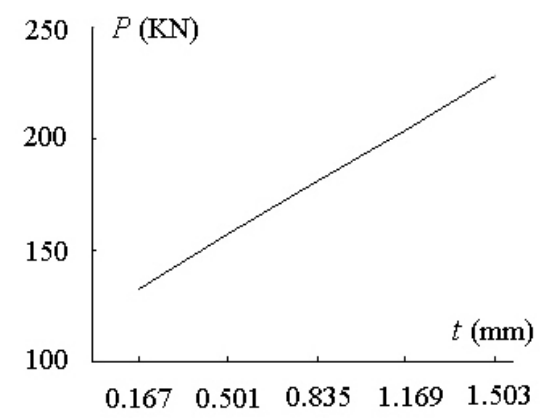

Fig.3 Effects of composites thickness on ultimate load

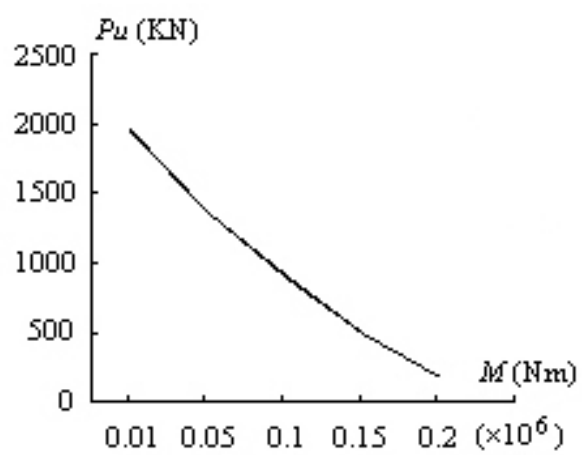

Fig.4 Comparison of analytical solutions and finite element solutions of ultimate loads (after pasting carbon fiber) 


\section{Conclusions}

(1) The theoretical formula in this paper can accurately calculate the ultimate load of buckling about neutral axis with maximum moment of inertia for perfect elastic-plastic rectangular column pasted with composites. The study have a very good reference value to the theoretical research of steel member reinforced by composites.

(2) The calculation example shows that the member reinforced by composites can obviously improve the behaviour of compression member that in plastic state and increase the plastic buckling bearing capacity of member. The stiffness of the member reinforced by composites is almost unchanged, the elastic core height of member is markedly increased, it indicates that steel member pasted with composites can greatly enhance the strength margin of member.

The plastoelastic instability formula of member with plastic zone both in compression-side and tension side will be researched in another paper.

\section{Acknowledgements}

This work was financially supported by The National Natural Science Fund (51678101).

\section{References}

[1] Miller Trent C, Chajes Michael J. Strengthening of a steel bridge girder using CFRP plates. Journal of Bridge Engineering, 2001, 6(6): 514-522

[2] Sen R, Liby L. Strengthening steel bridge sections using CFRP laminates. Composites Part B: Engineering, 2001, 32(4): 309-322

[3] Xinming Cao , Xiaoyun Xi. Influence of longitudinal steel on ductility of square columns under axial load. Journal of gaizhou university of technology.2002，31(5): 68-73.(In Chinese)

[4] Ling Zhao, Aiqun Li. Strength of circular concrete columns under concentric compression. Journal of Southeast University.2003,19(1):74-79.(In Chinese)

[5] Tavakkolizadeh M, Saadatmanesh H. Fatigue strength of steel girders strengthened with carbon fiber reinforced polymer patch. Journal of Structural Engineering, 2003, 129(2): 186-196 